\title{
THRESHOLD LOGIC: FROM VACUUM TUBES TO NANOELECTRONICS Invited Paper
}

\author{
Valeriu Beiu ${ }^{1}$, José M. Quintana ${ }^{2}$, María J. Avedillo ${ }^{2}$, and Mawahib Sulieman ${ }^{1}$ \\ ${ }^{1}$ School of Electrical Engineering and Computer Science, Washington State University \\ 102 Spokane Street, P.O. Box 642752, Pullman, WA 99164-2752 USA \\ ${ }^{2}$ Instituto de Microelectrónica de Sevilla - Centro Nacional de Microelectrónica (IMSE-CNM), \\ Universty of Sevilla, Edificio CICA, Avda. Reina Mercedes s/n, 41012-Sevilla, Spain
}

\begin{abstract}
This paper reviews silicon implementations of threshold logic gates, covering several decades. It details numerous VLSI implementations including: capacitive (switched capacitor and floating gate with their variations), conductance/current (pseudo-nMOS, output-wired-inverters, and a plethora of solutions evolved from them), as well as many differential solutions. Nanoelectronic implementations (e.g., based on negative resistance devices and on single electron technologies) will shortly be mentioned.
\end{abstract}

\section{INTRODUCTION}

Research on neural networks (NNs) goes back sixty years ago. The seminal year for the development of the "science of mind" was 1943 when the first mathematical model of a neuron operating in an all-or-none fashion: the threshold logic (TL) gate (TLG) was invented [2].

In the last decade the tremendous impetus of VLSI technology has made neurocomputer design a really lively research topic. Research on hardware implementations of NNs, and on TL in particular, has been very active. In this invited review paper we shall focus only on the many different approaches that have been tried for implementing $\mathrm{TL}$ in silicon. Effectiveness of TL as an alternative to modern VLSI design is determined by the availability, cost and capabilities of the basic building blocks. In this sense, many interesting circuit concepts for developing CMOS compatible TLGs have been explored. As the number of different proposed solutions reported in the literature is on the order of hundreds, we cannot mention all of them here. Instead, we shall try to cover important types of architectures and present several representative examples. For keeping the paper's length reasonable, the early days of
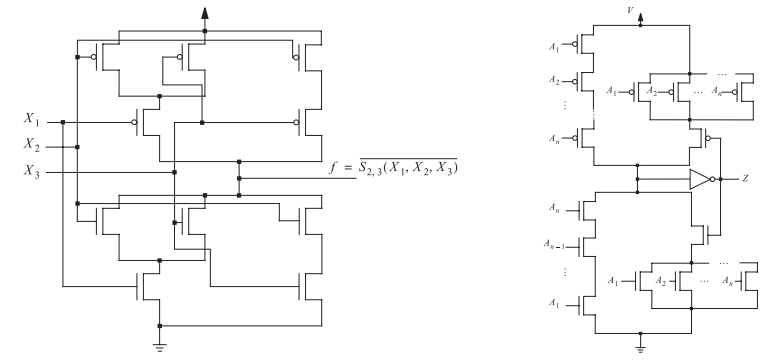

Figure 1

(a) CMOS solution for MAJORITY functions. (b) Static "NULL convention threshold logic" of an $n$-of- $n$ gate.
TL implementations (i.e., when the technologies were TTL, $\mathrm{ECL}, \mathrm{I}^{2} \mathrm{~L}$, and nMOS), although quite instructive, will not be covered here (the interested reader should consult [1]).

\section{CMOS SOLUTIONS}

Probably the first pure CMOS solution is due to Hampel [48] (Fig. 1 (a)). The CMOS devices form a plurality of TLG configurations having majority (MAJ) logic functions with near symmetrical switching delay times. MAJ functions are Threshold Logic Functions (TLFs) having identical weights. A MAJ gate can implement arbitrary TLFs by repeating/ complementing some of its inputs. The fact that the nMOS and pMOS stacks are alike leads to symmetrical switching delay times. This solution has low power consumption and large noise margins, but larger fan-in gates are slow.

A NULL Convention Logic gate [4] (Fig. 1(b)), receives a plurality of inputs, each having an asserted state and a NULL state. The TLG switches its output to an asserted state when the number of asserted inputs exceeds a threshold number. The TLG switches its output to the NULL state only after all inputs have returned to NULL. This is an asynchronous delay-insensitive design. The gate has low power and large noise margins, being reasonably fast for small fan-ins.

Another solution is based on pass-transistor logic. It offers an attractive alternative to pure CMOS. A steering circuit, which produces all the TLFs for an $n$-input logic function was detailed in [3]. A distinguishing characteristic is that pass-transistor-based ones depend only on the number of variables, not on their associated weights. This design inherits the problems specific to static pass-transistor logic.

\section{CAPACITIVE IMPLEMENTATIONS}

The concept underlying capacitive TLGs is the use of an array of capacitors to implement the weighted sum of the inputs. Capacitive threshold-logic gates can be classified into two major groups: Neuron MOS ( $v M O S)$, and Capacitive Threshold Logic (CTL). Although closely related, these two original approaches were different at the beginning: static versus clocked and different mechanisms for setting the threshold value, while their current developments have become increasingly similar. 


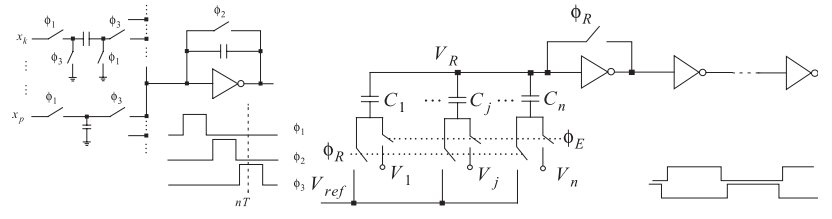

Figure 2

(a) Switched capacitor. (b) Capacitive threshold logic (CTL) gate.

\section{A. The Switched Capacitor}

Originally introduced in 1987 (Fig. 2 (a)) the main idea was to use switched capacitors, switches and inverters, and to take advantage of the inherent saturation of the inverters to implement the neuron non-linearity without additional elements [7]. This first approach required a somehow complex three-phase clock. The principle was also presented in [5]. It has quickly evolved into a simpler two-phase clock solution [6], known as the Capacitive Threshold Logic (CTL) gate (Fig. 2(b)). CTL gates have a regular structure, and are able to implement large fan-ins, while their main drawbacks are: large delays, large area, DC power consumption, and the threshold value programming mechanism. The reset time grows with the fan-in, and can become large. Propagation delay is logarithmic with the fanin, and has a strong dependence on the unit capacitor [6]. The area of the unit capacitor is equivalent to several minimum sized inverters. Due to the linear operation of the sense amplifier, the power consumption is high. Several solutions for overcoming CTL's limitations are detailed in [43].

\section{B. The Neuron-MOS Transistor}

The first CMOS capacitive solution was presented in 1966 [45] (Fig. 3 (a)). It was rediscovered 25 years later [14], when its integration led to the well-known neuron-MOS (vMOS): simple and compact. However, there are a number of problems: (i) degradation in the long-term stability (due to the floating gate); (ii) limited effective fan-in (due to sensitivity to parasitic charges and process variations); (iii) DC power consumption (for static $v$ MOS gates).

A solution for reducing the power dissipated by static
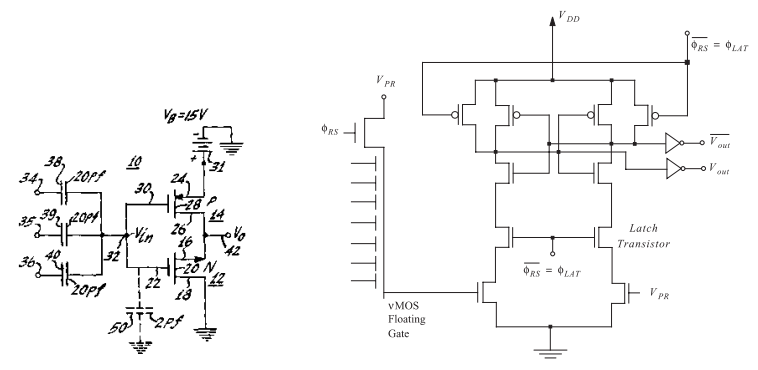

Figure 3

(a) First CMOS capacitive solution (b) Dynamic latched-sense-amplifier (comparator) neuron MOS.

vMOS TLGs is represented by the deep-threshold vMOS TLG [13]. Unfortunately, the penalty is an almost threefold increase in delay. In the clock-controlled vMOS TLG [12], a clock-driven switch is attached to the floating gate to initialise the floating-gate charge. A similar concept is used by the Controlled Floating-Gate Devices (CFGDs) [8]. These dynamic versions still have relatively high static power and might require multiple phase clocks. The static power consumption of the basic VMOS TLG can be eliminated, and its speed increased, by a current comparison between a $v$ MOS transistor and a reference device, using a positive feedback. Different configurations taking advantage of this concept have been reported. One example is the sense-amplifier $v$ MOS TL [12] (Fig. 3 (b)). A variation, called CMOS Capacitor Coupling Logic $\left(\mathrm{C}^{3} \mathrm{~L}\right)$, uses the capacitor coupling technique and a current sense amplifier [11] (Fig. 4 (a)). Another low-power solution is the Charge Recycling Threshold Logic (CRTL) gate [9] (Fig. 4 (b)). CRTL gates exhibit high-speeds, and are suitable for high fan-ins, while also having low power consumption. In fact, CRTL gates achieve the highest speed and $15-20 \%$ lower power consumption when compared with clocked vMOS [12], $C^{3} L$ [11], and LCTL [23]. Recently, a Self-Timed Threshold Logic (STTL) has been proposed [10] (Fig. 4 (c)). It describes a "capacitor sharing" technique for significantly reducing the occupied area, which can be applied to other $v$ MOS implementations. The self-timing idea comes from asynchronous circuits; eliminating the clock can reduce the power even more.

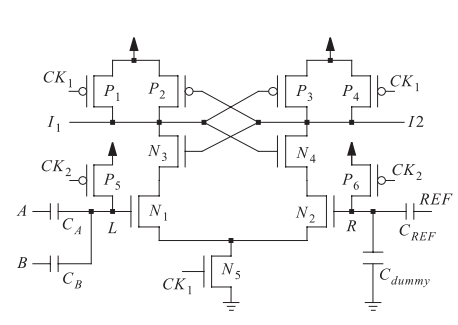

(a) CMOS capacitor coupling logic $\left(\mathrm{C}^{3} \mathrm{~L}\right)$;

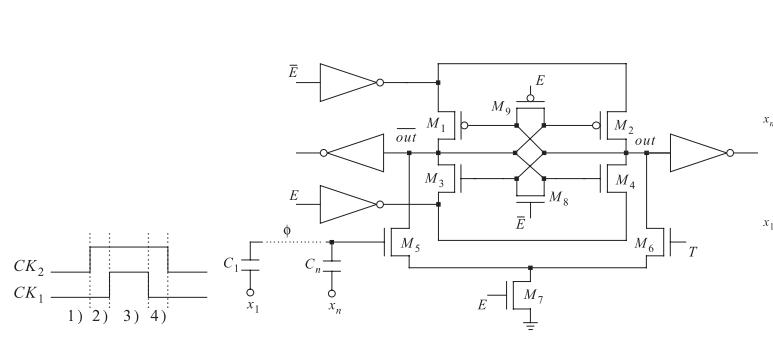

Figure 4

(b) Charge recycling threshold logic (CRTL); (c) Self-timed threshold logic (STTL), an asynchronous sense amplifier differential logic with self-timed enable signaling. 


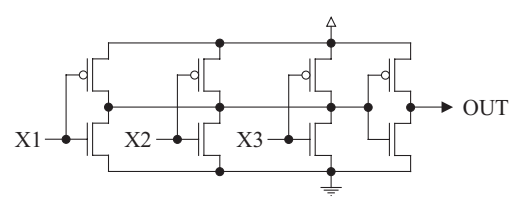

Figure 5

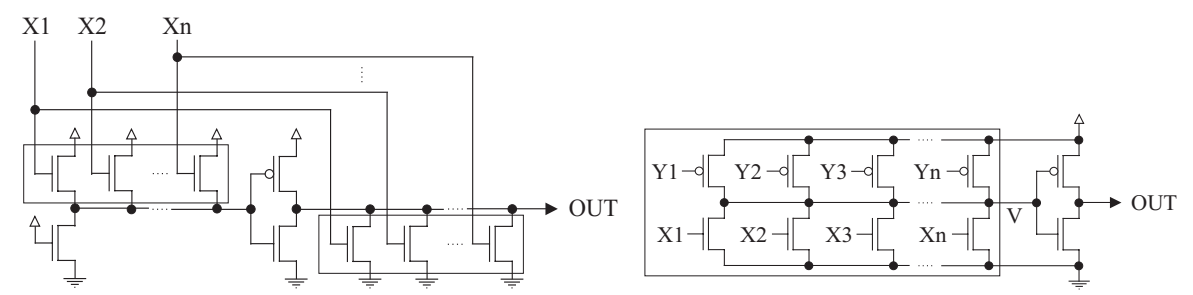

(a) Output wired inverters discovered by Lerch. (b) Source follower pull-up logic (SFPL). (c) Beta-driven threshold element ( $\beta$ DTE).

\section{CONDUCTANCE IMPLEMENTATIONS}

\section{A. Conductance / Current Solutions}

Two early CMOS TL solutions have been enduring: $(i)$ the pseudo-nMOS (also know as grounded-pMOS), and (ii) the output-wired-inverters (also known as ganged CMOS). These two solutions have represented the starting points of two long series of variations/modifications, which made incremental enhancements on: $(i)$ their DC power consumption, and ( $i$ i) their reduced noise margins.

The nMOS technology was suitable for high fan-in gates. A depletion nMOS transistor was used as a load, making NOR gates very fast. In CMOS, the solution was to use a pMOS with its gate grounded. This is the pseudonMOS solution: fast, having DC power, and using ratio rules. The reduced output voltage swing and gain makes the gate more susceptible to noise. Because the gate of the pullup pMOS transistor can be turned off, pseudo-nMOS can easily implement a power-down mechanism. Another advantage of such gates is their low transistor count. The ratio rules make it possible to implement TLFs. PseudonMOS TLG have reduced noise margins, as the common node has meaningful analogue voltages. That is why these gates are limited to small fan-ins, and an inverter is used for buffering and voltage recovering.

The second solution is based on inverters with their outputs hard-wired together, and was detailed in 1973 [46] (Fig. 5 (a)). Unfortunately, due to the sensitivity of the voltage on the common node, and of the $V_{t h}$ of the output inverter to process variations, the output-wired-inverter TLGs are fan-in limited. These TLGs are extremely fast, while exhibiting high power consumption (assumable when traded-off for speed), as well as narrow noise margins. The output-wired-inverters TLGs have been rediscovered several times. In [44] a very fast NOR gate was presented: it is Lerch's construction [46] without the restoring inverter. This gate was used in the MIPS R2010 [44]. Later, Schultz et al. [18] rediscovered Lerch's original construction, and called it "Ganged-CMOS logic" (GCMOS) [47].

\section{B. Beyond Pseudo-nMOS}

A lot of effort has been devoted to reducing the power consumption of large fan-in pseudo-nMOS gates. The other drawback - the reduced noise margins - was left as an open question. The main idea for reducing the DC power was to replace the pMOS load with a more complex load circuit. Such solutions rely on: using asynchronous feedback and/or feedforward, reducing the voltage swings, using a clock signal (dynamic solutions), using controlled current mirrors, or even data-dependent solutions (for details see [43]).

\section{Beyond Output-Wired-Inverters}

Output-wired-inverters suffer from the same disadvantages as pseudo-nMOS solutions: DC power consumption and reduced noise margins. A first enhancement was to connect the inputs only to the nMOS transistors. This increases the speed but does not improve on the power consumption or on the noise margins. A modification to the basic idea was introduced in [20] (Fig. 5 (b)), where a new class of logic gates called Source Follower Pull-up Logic (SFPL) is described (an enhanced version was used in the Sun Sparc). The power dissipation is large, while the noise margins are acceptable. A systematic method for designing TLGs having only one transistor per input, led to the $\beta$-Driven Threshold Element ( $\beta$ DTE) [21] (Fig. 5 (c)). The $\beta$ DTE reduces the input and the internal node capacitance, making the gate very fast, but does not tackle either the power consumption or the narrow noise margins. An improved $\beta$-comparator having higher non-linearity in the threshold zone was presented in [22]. A simpler method for enhancing the noise margins is presented in [19] (Fig. 6 (a)). It adds datadependent non-linear terms to the $\beta$ DTEs, converting the TLG into a "high order perceptron." The non-linear terms form a Noise Suppression Logic (NSL). For reducing the DC power a data-dependent Self-Timed Power-Down

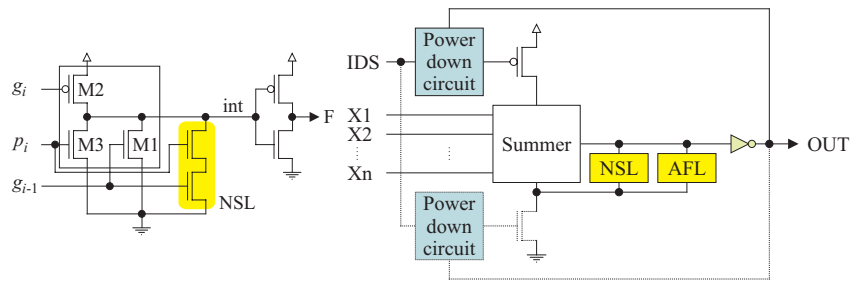

Figure 6

(a) Implementation with noise suppression logic (NSL).

(b) Data-dependent self-time power-down (STPD) mechanism. 


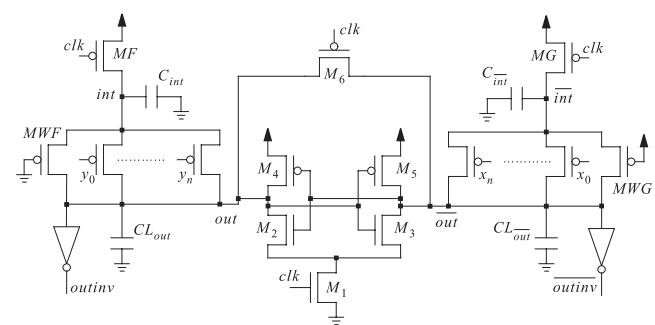

Figure 7

(a) Digital comparators based on cross-coupled inverters with asymmetrical loads (CIAL). (b) Latch type threshold logic (LCTL).

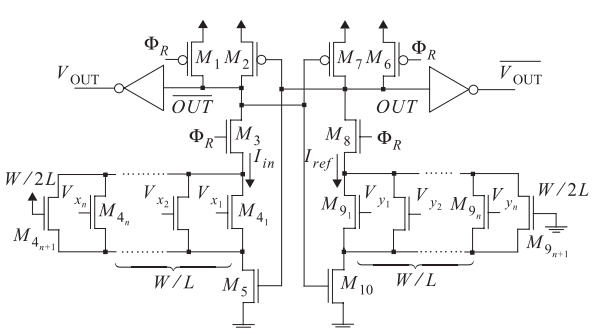

mechanism (STPD) has also been developed [19] (Fig. 6 (b)).

\section{Differential Solutions}

Differential solutions have also been investigated due to their very low power consumption (as eliminating DC power).

The first differential solution based on resistor-diode structures and bipolar transistors was introduced in 1964 [49]. The operation of Cross-coupled Inverters with Asymmetrical Loads (CIAL) was exploited to implement comparators [28], a particular example of a TLG (Fig. 7 (a)). At the same time, a generic Latch-Type TL (LCTL) gate was proposed in [23] (Fig. 7 (b)). The speed performance of LCTL gates has been improved in [26]. It is called Crosscouple Inverters with Asymmetrical Loads Threshold Logic (CIALTL), and can be seen in Fig. 8 (a). Recently, a number of solutions based on advanced clocked CMOS differential structures have been developed. These implement the pulldown networks with two banks of parallel nMOS transistors, instead of using nMOS complementary logic trees. Examples are: Single-input Current-Sensing Differential Logic (SCSDL) [30], Differential Current-Switch Threshold Logic (DCSTL) [24] (Fig. 8 (b)) after the DifferentialCurrent Switch Logic (DCSL) [29], and Current-Mode Threshold Logic (CMTL) [25]. These TLGs are still sensitive to noise and mismatch of process parameters, which limit their maximum fan-in. Yield analysis for SCSDL have shown that fan-in $\leq 14$ [30]. An enhancement is to implement $f$ with one bank and $f$ ' with the other bank, and include an NSL both for $f$ and $f^{\prime}$ [31]. The fact that $f$ and $f^{\prime}$ always have transitions in opposite directions leads to increased speed and better noise margins. This method was demonstrated in conjunction with the Split-level Precharge Differential (SLPD) logic [27] (see [31] and Fig. 9), but can be used with any differential solution.

\section{OTHER IMPLEMENTATIONS}

Many other approaches have been used for implementing TLG: Josephson junctions, charge-coupled devices, optical, and even molecular. The emerging future devices are: single electron devices, RTDs, double layer tunneling transistors, and Schottky barrier MOSFET.

\section{A. Solutions Single Electron Tunneling (SET) Solutions}

SET has been receiving increased attention because it combines large integration and ultra low power dissipation. Recently, the use of SET technology for TLCs has been advocated, and several implementations have been presented. They use a capacitor array for input summation, similar to the solutions reported in Section III.B, but differ in the way the thresholding operation is performed. A SET implementation of MAJ gates, similar to the static $\mathrm{MOOS}$, is presented in [32] (Fig. 10 (a)). Another $n$-input TLG was proposed in [33]. It requires one tunnel junction and $n+2$ true capacitors (Fig. 10 (b)). A MAJ gate using a balanced pair of single electron boxes has also been detailed [34]. A parallel prefix 16-bit adder designed using capacitive input SETs has been recently characterized [35].

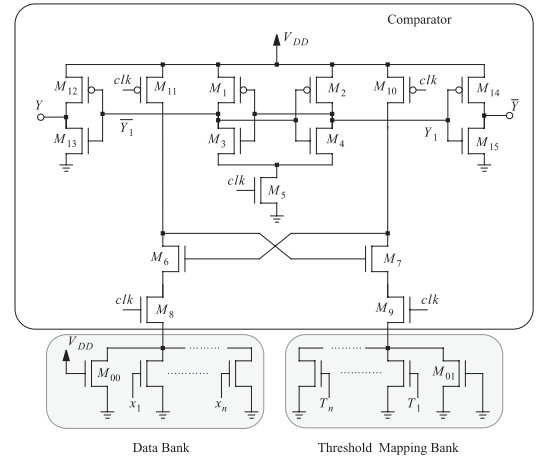

Figure 8

(a) Cross-couple inverters with asymmetrical loads threshold logic (CIALTL). (b) Differential current-switch threshold logic DCSTL. 


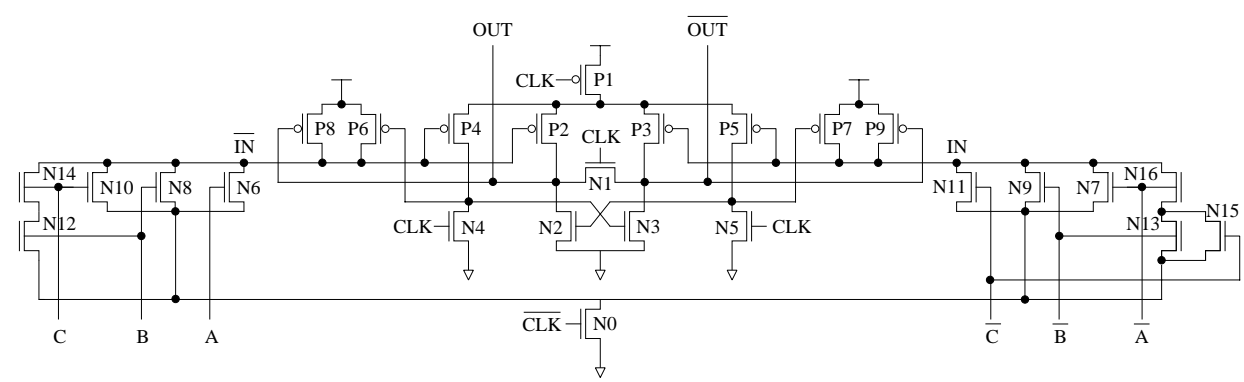

Figure 9

Split-precharge differential noise-immune threshold logic (SPD-NTL)

\section{B. Resonant Tunneling Devices (RTDs)}

NRDs have been proposed for implementing TLGs as early as 1961 [50] (see also [40]). While RTD is the basic twoterminal negative differential resistance (NDR) device, it is also possible to introduce tunneling within conventional bipolar devices. Hence three-terminal NDR devices, such as Resonant Tunneling Bipolar Transistors (RTBTs) or Resonant HETs (RHETs) can be obtained. Circuit applications of RTDs are mainly based on the MOnostableBIstable Logic Element (MOBILE) [36] (Fig. 11 (a)). A specific logic functionality of a MOBILE is determined by embedding an input stage, which modifies the peak current of one of the RTDs. TLGs implemented with RTDs have been widely studied [37]. Other configurations are possible, but the major advantage comes from the fact that the NRD characteristic directly supports multiple valued logic style [41], making TL an ideal candidate (Fig. 11 (b)).

RTDs are the most mature type of quantum-effect devices. They exhibit NDR at room temperature, and have been already implemented [39]. A prototyping technique based on a four MOS-NDR transistors has also been reported [38].

\section{CONCLUSIONS}

The present state-of-the-art shows a large variety of TLG implementations coping with their major drawbacks: power dissipation, reduced noise margins, and the sensitivity to process variations. It is quite amazing how much effort and ingenuity has been spent/invested, let alone the remarkable diversity of technologies that have been tried. TLGs have
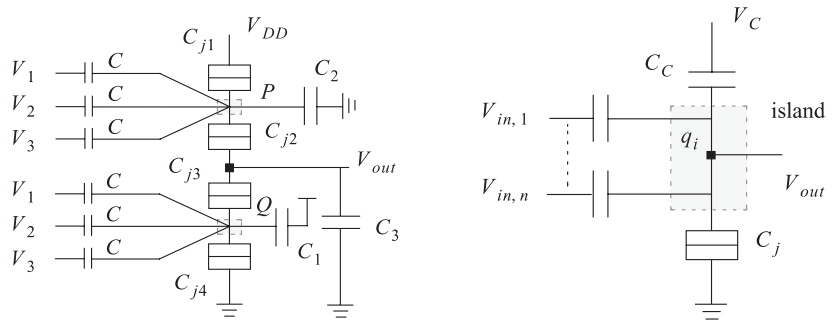

Figure 10

(a) Three-input MAJORITY gate. (b) TLG with one tunnel junction. benefited from developments in the more general field of differential logic. The other design parameter to consider is the fan-in. The claim that TLG should have a large fan-in comes from their original goal of mimicking the brain, but theoretical results [42] have shown that small fan-ins can lead to VLSI-optimal solutions. The scarcity of commercial applications is not because TLGs have poor performances. Advanced TLGs can easily compete with BGs. So why are they not used? The answer has its roots in the design approach, namely the fact that TLGs need full custom design and that there is an acute lack of high-level synthesis tools.

Lastly, because nano computing will probably get central stage positions in the (near) future, TL will surely benefit from that. Both RTDs and SET appear to hold the most promise as a short to medium-term solution. The fact that TL is a perfect fit for them will certainly help their future developments.

\section{ACKNOWLEDGMENT}

V. Beiu and M. Sulieman were partly sponsored by the Air Force Research Laboratory under agreement number F29601-02-2-0299. The U.S. Government is authorized to reproduce and distribute reprints for Governmental purposes notwithstanding any copyright notation thereon. The views and conclusions contained herein are those of the author and should not be interpreted as necessarily representing the official policies or endorsements, either expressed or implied, of the Air Force Research Laboratory or the U.S. Government. J.M. Quintana and M.J. Avedillo were partly sponsored by EU under IST 2001-32358 Project "QUDOS."

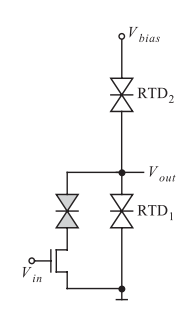




\section{REFERENCES}

[1] V. Beiu, "Threshold logic implementations: The early days," Proc. MWSCAS'03, Cairo (Egypt), 2003 (this proceedings).

[2] W.S. McCulloch, W. Pitts, "A logical calculus of the ideas immanent in nervous activity," Bull. Math. Biophysiol., 5, pp. 115-133, 1943.

[3] J.M. Quintana, M.J. Avedillo, R. Jiménez, E. Rodríguez-Villegas, "Practical low-cost CPL implementations of threshold logic functions," Proc. GLSVLSI'01, West Lafayette (USA), 2001, pp. 139144.

[4] G.E. Sobelman, K. Fant, "CMOS circuit design of threshold gates with hysteresis”, Proc. ISCAS’98, Monterey (USA), 1998, pp. 61-64.

[5] U. Çilingiroğlu, "A purely capacitive synaptic matrix for fixed-weight neural networks," IEEE Trans. Circ. Syst. II, 38, 210-217, 1991.

[6] H. Özdemir, A. Kepkep, B. Pamir, Y. Leblebici, U. Çilingiroğlu, "A capacitive threshold-logic gate," IEEE J. Solid-State Circ., 31, pp. 1141-1150, 1996.

[7] Y.P. Tsividis, D. Anastassiou, "Switched-capacitor neural networks," Electron. Lett., 23, pp. 958-959, 1987.

[8] K. Aoyama, "A reconfigurable logic circuit based on threshold elements with a controlled floating gate," Proc. ISCAS'02, Scottsdale (USA), 2002, Vol. 5, pp. 381-384.

[9] P. Celinski, J.F. López, S. Al-Sarawi, D. Abbott, "Low power, high speed, charge recycling CMOS threshold logic gate," Electron. Lett., 37, pp. 1067-1069, 2001.

[10] P. Celinski, J.F. López, S. Al-Sarawi, D. Abbott, "Compact parallel $(m, n)$ counters based on self-timed threshold logic", Electron. Lett., 38, pp. 633-635, 2002.

[11] H.Y. Huang, T.N. Wang, "CMOS capacitor coupling logic $\left(\mathrm{C}^{3} \mathrm{~L}\right)$ logic circuits," Proc. AP-ASIC'00, Cheju (Korea), 2000, pp. 33-36.

[12] K. Kotani, T. Shibata, M. Imai, T. Ohmi, "Clocked-controlled neuronMOS logic gates,” IEEE Trans. Circ. Syst. II, 45, pp. 518-522, 1998.

[13] H.-Y. Kwon, K. Kotani, T. Shibata, T. Ohmi, "Low power neuronMOS technology for high-functionality logic gate synthesis", IEICE Trans. Electr., E80-C, pp. 924-929, 1997.

[14] T. Shibata, T. Ohmi, "A functional MOS transistor featuring gatelevel weighted sum and threshold operations," IEEE J. Solid-State Circ., 39, pp. 1444-1455, 1992.

[15] W. Weber, S.J. Prange, R. Thewes, E. Wohlrab, A. Luck, "On the application of neuron MOS transistor principle for modern VLSI design," IEEE Trans. Electr. Dev., 43, pp. 1700-1708, 1996.

[16] J.M. Quintana, M.J. Avedillo, A. Rueda, and S. Baena, "Hazard-free edge-triggered D flipflop based on threshold gates," Electr. Lett., 30, pp. 1390-1391, 1994.

[17] J.M. Quintana, M.J. Avedillo, A. Rueda, C. Baena, "Practical low-cost CMOS realization of complex logic functions, "Proc. ECCTD'95, Istanbul (Turkey), 1995, pp. 51-54.

[18] K.J. Schultz, R.J. Francis, K.C. Smith, "Ganged CMOS: trading standby power for speed," IEEE J. Solid-State Circ., 25, pp. 870-873, 1990.

[19] V. Beiu, "On higher order noise immune perceptrons," Proc. IJCNN'01, Washington (USA), 2001, pp. 246-251.

[20] T.D. Simon, "A fast static CMOS NOR gate," Proc. Brown/MIT Conf. $A d v$. Res. VLSI \& Parallel Sys. (T. Knight, J. Savage, Eds.), Cambridge, MA: MIT Press, 1992, pp. 180-192.

[21] V.I. Varshavsky, " CMOS artificial neuron on the base of $\beta$-driven threshold element," Proc. SMC'98, San Diego (USA), Vol. 2, 1998, pp. 1857-1861.

[22] V.I. Varshavsky, and V. Marakhovsky, " $\beta$-CMOS artificial neuron and implementability limits," Proc. IWANN'99, Alicante (Spain), Vol. 2, 1999, pp. $117-128$.

[23] M.J. Avedillo, J.M. Quintana, A. Rueda, E. Jiménez, “A low-power CMOS threshold-gate," Electr. Lett., 31, 2157-2159, pp. 1995.

[24] M. Pădure, S. Coțofană, C. Dan, M. Bodea, S. Vassiliadis, "A new latch-based threshold logic family," Proc. CAS'01, Sinaia (Romania), 2001, Vol. 2, pp. 531-534.

[25] S. Bobba, I.N. Hajj, "Current-mode threshold logic gates," Proc. ICCD'00, Austin (USA), 2000, pp. 235-240.
[26] J.F. Ramos, J.A.H. López, M.J. Martín, J.C. Tejero, A.G. Bohórquez, "A threshold logic gate based on clocked coupled inverters," Intl. J. Electr., 84, 371-382, pp. 1998.

[27] J. Lee, J. Park, B. Song, W. Kim, "Split-level precharge differential logic: A new type of high-speed charge-recycling differential logic," IEEE J. Solid-State Circ., 36, pp. 1276-1280, 2001.

[28] J.A.H. López, J.G. Tejero, J.F. Ramos, A.G. Bohórquez, "New types of digital comparators," Proc. ISCAS'95, Seattle (USA), Vol. 1, 1995, pp. 29-32.

[29] D. Somasekhar, K. Roy, "Differential current switch logic: A low DCVS logic family," IEEE J. Solid-State Circ., 31, pp. 981-991, 1996.

[30] R. Strandberg, J. Yuan, "Single input current-sensing differential logic (SCSDL)," Proc. ISCAS'00, Geneva (Switzerland), 2000, Vol. 1, pp. 764-767.

[31] S. Tatapudi, V. Beiu, "Split-precharge differential noise-immune threshold logic gate (SPD-NTL)," Proc. IWANN'03, Menorca (Spain), 2003, Vol. 2, pp. 49-56.

[32] H. Iwamura, M. Akazawa, Y. Amemiya, "Single-electron majoriy logic circuits," IEICE Trans. Electr., E-81C, pp. 42-48, 1998.

[33] C. Lageweg, S. Coțofană, S. Vassiliadis, "A linear threshold gate implemented in single electron technology," Proc. WVLSI'01, Orlando (Florida), 2001, pp. 93-98

[34] T. Oya, T. Asai, T. Fukui, I. Ameniyaa, "A majority-logic nanodevice using a balance pair of single-electron boxes," J. Nanosci. Nanotech., 2, pp. 333-342, 2002.

[35] M. Sulieman, V. Beiu, "Characterization of a 16-bit threshold logic single electron adder," Proc. ISCAS'04, Vancouver (Canada), May 2004 , to appear.

[36] T. Akeyoshi, K. Maezawa, T. Mizutani, "Weighted sum threshold logic operation of MOBILE's (monostable-bistable transition logic element) using resonant-tunneling transistors," IEEE Electr. Dev. Lett., 14, pp. 475-477, 1993.

[37] M. Bhattacharya, P. Mazumder, "Noise margins of threshold gates containing resonant tunneling diodes," IEEE Trans. Circ. Syst. II, 47, pp. $1080-1085,2000$.

[38] A.F. González, M. Bhattacharya, S. Kulkarni, P. Mazumder, "CMOS implementation of a multiple-valued logic signed-digit full adder based on negative-differential-resistance devices," IEEE J. Solid-State Circ., 36, pp. 924-932, 2001.

[39] T. Itoh, T. Waho, K. Maezawa, M. Yamamoto, "10-GHz operation of multiple-valued quantizers using resonant-tunneling devices," IEICE Trans. Info. Sys., E82-D, pp. 949-954, 1999.

[40] C. Pacha, U. Auer, C. Burwick, P. Glösekötter, A. Brennemann, W. Prost, F.-J. Tegude, K.F. Goser, "Threshold logic circuit design for parallel adders using resonant tunneling devices," IEEE Trans. VLSI Sys., 8, pp. 558-572, 2000.

[41] T. Waho, "Resonant tunneling transistor and its application to multiple-valued logic circuits," Proc. ISMVL'95, Bloomington (USA), 1995, pp. 130-138.

[42] V. Beiu, H.E. Makaruk, "Deeper sparser nets can be optimal," Neural Proc. Lett., 8, pp. 201-210, 1998.

[43] V. Beiu, J. Quintana and M. Avedillo, "VLSI implementation of threshold logic: A comprehensive survey," IEEE Trans. Neural Networks, Special Issue on Hardware Impl., 14, pp. 1217-1243, 2003.

[44] M.G. Johnson, "A symmetric CMOS NOR gate for high-speed applications," IEEE J. Solid-State Circ., 23, pp. 1233-1236, 1988.

[45] J.R. Burns, "Threshold circuit utilizing field effect transistors," U.S. Patent 3260 863, Jul. 12, 1966.

[46] J.B. Lerch, "Threshold gate circuits employing field-effect transistors," U.S. Patent 3715 603, Feb. 6, 1973.

[47] K.J. Schultz, and K.C. Smith, "A CMOS binary adder using quaternary ganged-logic internal node," Proc. ISMVL'89, Guangzhou (China), 1989, pp. 356-359.

[48] D. Hampel, K.J. Prost, and N.R. Scheinberg, "Threshold logic using complementary MOS device," U.S. Patent 3900 742, Aug. 19, 1975.

[49] V.J. Modiano, "Majority circuit using a constant current bias," U.S. Patent 3155 839, Nov. 3, 1964.

[50] T.B. Tomlinson, "Non-linear resistance devices," U.S. Patent 3011075 , Nov. 28,1961 